\title{
Partizipatorische pädagogische Diagnostik
}

\section{Ein Modell für mehr Selbstbestimmung aller Schülerlnnen bei Lernprozessen und Bildungswegentscheidungen}

\author{
Sabine Gerhartz-Reiter \& Cathrin Reisenauer
}

Journal für Psychologie, 26(2), 114-132

https://doi.org/10.30820/8248.07

www.journal-fuer-psychologie.de

\section{Zusammenfassung}

Lehrpersonen haben im Rahmen der pädagogischen Diagnostik neben der Analyse von Lernvoraussetzungen und Lehr-Lernprozessen den Auftrag, »Zuweisung zu Lerngruppen oder zu individuellen Förderungsprogrammen [zu] ermöglichen « (Ingenkamp \& Lissmann, 2008, S. 13). Den damit verbundenen Zuschreibungsprozessen, die einen »Ort der Macht « (Butler, 2009, S. 11) darstellen, wohnt ein identitätsstiftender und transformierender Charakter inne. Für SchülerInnen spielt pädagogische Diagnostik damit eine zentrale Rolle für ihr Selbstkonzept, ihre Bildungs- und infolge ihre Berufs- und Lebenschancen. Im Zentrum dieses Beitrags wird über ein Forschungsprojekt berichtet, das die Frage verfolgt, wie SchülerInnen erkennen und deutlich machen können, was sie brauchen, um zu werden, was sie werden können und wollen, und nicht durch das Zuschreiben oder Absprechen von Fähigkeiten beschränkt werden. Die konzipierte partizipatorische Diagnostik bietet ein Modell, mithilfe dessen Lehrpersonen alle SchülerInnen auf ihrer Entwicklung hin zu aktiven GestalterInnen ihrer Bildungsprozesse begleiten können, und dient auch dazu, vorhandene Barrieren, gesellschaftliche Normen und Normalitätsvorstellungen sowie wirkmächtige Diskurse und Machtverhältnisse sichtbar zu machen.

Schlüsselwörter: Pädagogische Diagnostik, partizipative Forschung, Partizipation, Disability Studies, Bildungskarrieren

\section{Summary}

Participatory pedagogical diagnostics. A model for more self-determination in all students' learning processes and educational careers

Teachers play a significant role for their students' educational careers. Besides analysis of conditions of learning and learning processes, the field of pedagogical diagnostics implies the allocation of students to groups of learners or to special support programs (Ingenkamp \& Lissmann, 2008). Consequently, pedagogical diagnostics are crucial for the students' selfconcept, their prospective educational opportunities and thus also for their career and life 
opportunities. Therefore, the fact that students' knowledge concerning their abilities and needs are hardly used in the process of diagnostics needs to be changed. This paper deals with the question how students themselves can recognise and make clear what they are able to do and what they need in order to become what they want to become and not be restricted by others' attributions of (non-)ability. The presented approach of emancipatory and dialogic diagnostics enables all students to actively design their educational processes. Moreover, it helps to make existing barriers, social norms and normative notions of reality as well as efficacious discourses and power structures visible in order to contribute to their reduction.

Keywords: pedagogical diagnostics, participatory research, disability studies, educational careers

\section{Einleitung}

Lehrkräfte nehmen auf vielfältige Weise Einfluss auf ihre SchülerInnen. Ein wesentlicher Aspekt dabei ist der Bereich der pädagogischen Diagnostik, der vielfach als eines der zentralen Aufgabenfelder von Lehrpersonen bezeichnet wird (u. a. OECD, 2012). Auf Basis von Beobachtungen und Leistungsfeststellungen durch Lehrkräfte sind SchülerInnen, ihre Leistungen, lernbezogenen Verhaltensweisen und Bedürfnisse Objekte eines laufenden, einflussreichen Diagnoseprozesses. Oftmals wird jedoch die mangelnde »Urteilsakkuratheit « der Diagnosen, die in der Regel höher ist, je mehr Quellen miteinbezogen werden, kritisiert (van Ophuysen \& Behrmann, 2015, S. 83). Die SchülerInnen selbst in der Regel nicht oder kaum in diesen Prozess einzubinden bedeutet, dass fruchtbare Ressourcen für die Diagnose nicht genutzt werden, die ein umfassenderes Bild der Leistungen bzw. Leistungspotenziale sowie Bedürfnisse der Kinder und Jugendlichen zeichnen und eine geringere Abhängigkeit der Diagnosen von Werten, Erwartungen und soziokultureller Prägung der Lehrkraft ermöglichen würden. Dies ist von hoher Relevanz insbesondere unter Berücksichtigung der Tatsache, dass Diagnosen der Lehrkräfte »das Leben des Kindes langfristig und in vielen Bereichen - vom aktuellen Wohlbefinden bis zum späteren beruflichen Erfolg - beeinflussen kann « (ebd., S. 87; vgl. auch Ingenkamp \& Lissmann, 2008, S. 13).

Darüber hinaus birgt eine pädagogische Diagnostik ohne Einbindung der SchülerInnen das Risiko einer großen Ohnmacht aufseiten der Kinder und Jugendlichen: Diagnostik wird hier als Urteil über die eigene Person wahrgenommen, auf das man selbst keinen Einfluss hat. Die häufig mit der Diagnose verbundenen Zuschreibungen von (Un-)Fähigkeiten bzw. (besonderen) Bedürfnissen prägen das Selbstkonzept der SchülerInnen und beeinflussen ihre weiteren Bildungsverläufe. 
Auf Basis der Ergebnisse von zwei unabhängigen Studien, mit denen einerseits die Rolle und Relevanz des LehrerInnenhandelns für gelingende Bildungskarrieren erklärt (Gerhartz-Reiter, 2017) ${ }^{1}$ und andererseits die Bedeutung sowie der transformierende Charakter von Anerkennung im pädagogischen Handeln aufgezeigt (Reisenauer \& Ulseß-Schurda, 2018) wurden, stellt sich die Frage, wie alle SchülerInnen - unabhängig von ihren individuellen Voraussetzungen - nicht nur eine deutlich aktivere Rolle in Bezug auf ihre Schul- und Bildungslaufbahnen einnehmen können, sondern ihnen darüber hinaus eine Emanzipation von Fremdurteilen und Zuschreibungen ermöglicht werden kann. Ziel ist es, Kinder aus einer oftmals vorhandenen Ohnmacht und einem Ausgeliefert-Sein in eine Teilhabemöglichkeit und Selbstbestimmung zu begleiten.

Grundlage für das vorliegende Projekt, das sich in den Disability Studies verortet, sind eine Sicht von »Behinderung als soziale[r] Konstruktion « sowie das Ziel, $\gg$ Prozesse zu untersuch[en], die zum gesellschaftlichen Ausschluss von Menschen mit Beeinträchtigung - eben zur Behinderung - führen « (Pfahl \& Köbsell, 2014, S. 554). Ziel ist es, ein Konzept im Rahmen pädagogischer Diagnostik zu entwickeln und zu etablieren, das darauf fokussiert, alle SchülerInnen in der Wahrnehmung und der Entfaltung ihrer Potenziale zu unterstützen, um Einschränkungen und ein »BehindertWerden « aufgrund von einseitigen diagnostischen Zuschreibungen zu vermeiden. Bei der Entwicklung eines Modells partizipatorischer Diagnostik ist zentral, dass diese Forschung mit Kindern und nicht über sie erfolgt und damit an ihren realen Erfahrungen ansetzt.

Im Folgenden werden zunächst zentrale Ergebnisse und Erkenntnisse aus den Studien vorgestellt, auf denen der vorliegende Artikel aufbaut: Nach der Erläuterung der Rolle von Lehrpersonen für die Subjektwerdung ihrer SchülerInnen werden Chancen und Risiken des LehrerInnenhandelns für Bildungskarrieren und Implikationen für die pädagogische Diagnostik dargestellt. Nach einem Überblick über aktuelle Formen der und Erkenntnisse zur pädagogischen Diagnostik folgt eine Erläuterung der Notwendigkeit eines neuen Ansatzes, der die Rolle aller SchülerInnen stärkt. Anschließend wird das von den Autorinnen entwickelte Konzept einer partizipatorischen Diagnostik erläutert, das für alle Lernenden unabhängig von ihren Grundvoraussetzungen umsetzbar sein soll, bevor Limitationen des Modells aufgezeigt und Schlussfolgerungen für die pädagogische Praxis gezogen werden.

\section{Theoretische und empirische Basis}

Lehrpersonen stellen wichtige AkteurInnen im schulischen Feld dar. Ihren Kompetenzen und ihrem Handeln wird große Bedeutung eingeräumt, da sie »einen erheblichen Einfluss auf die Lernentwicklung von Schülern « haben (Lipowski, 2006, S. 64). Leh- 
rerInnenhandeln beinhaltet großes Potenzial, aber auch »eine gewisse Riskanz für die SchülerInnen « (Helsper, 2000, S. 152), denn es kann langfristige Auswirkungen auf deren Subjektwerdung und Bildungsverläufe haben. Schule als Sozialisationsinstanz ist ein Ort, an dem sich die Entwicklung der ihr anvertrauten Kinder und Jugendlichen vollzieht und LehrerInnen dabei pädagogisch tätig sind. Pädagogisches Handeln zeichnet sich nach Ricken (2009, S.87) dadurch aus, dass »LehrerInnen SchülerInnen in bestimmter Weise ansprechen und adressieren, darin diese als jemanden wahrnehmen und zu jemandem machen «. Dies trifft in besonderer Weise auf die pädagogische Diagnostik zu, die den Auftrag hat, Lernvoraussetzungen und Lernprozesse zu analysieren und SchülerInnen Lerngruppen oder Förderprogrammen zuzuweisen und damit Bildungswege zu steuern (Ingenkamp \& Lissmann, 2008, S. 13). SchülerInnen werden in diesem Prozess von ihren LehrerInnen beispielsweise als fähig bzw. eben nicht fähig, als kompetent oder nicht so kompetent adressiert. LehrerInnen beeinflussen durch ihre Diagnosen und die darauf aufbauenden Adressierungen und pädagogischen Weichenstellungen die Selbstwahrnehmung und das Selbstkonzept der Kinder und Jugendlichen. Individuen entwickeln sich in und durch Beziehungen zu anderen Subjekten und sind somit nicht als per se autonom, sondern in Abhängigkeit von anderen zu verstehen (vgl. Hegel, 1970 [1806/07]). Lehrende stellen als pädagogisch Handelnde ein signifikantes Gegenüber für die Subjektwerdung und das Lernen von SchülerInnen dar. Die Studie von Reisenauer und Ulseß-Schurda (2018) zeigt, welche Anlässe und Abläufe Adressierungen von SchülerInnen durch ihre LehrerInnen haben und wie sich diese Adressierungen auf das Lernen, die Entwicklung, das Selbstverständnis und die Subjektwerdung von Kindern und Jugendlichen auswirken. Ausgehend von der Annahme, dass » man [...] nicht erst jemand [ist], der dann auf andere stößt, sondern [...] erst jemand durch andere und von anderen her [wird], ohne dass man deswegen vorher niemand war « (Balzer \& Ricken, 2010, S. 63), spielen Lehrende, deren pädagogisches Handeln und Diagnostizieren eine zentrale Rolle.

Judith Butler (2003) sieht Adressierungen - als wer jemand von wem für was und als was angesprochen wird - als Medium, in dem sich die Subjektwerdung von Menschen vollzieht. »[D]ass das Subjekt diskursiv konstituiert ist «, zeigt sich auf die Schule bezogen besonders darin, dass sich Subjekte durch pädagogische Adressierungen, wie sie eben auch diagnostische Urteile darstellen, nicht nur entwickeln, sondern in besonderem Maße hervorgebracht werden. So wird beispielsweise durch die Feststellung eines sonderpädagogischen Förderbedarfes erst eine Identität als »SonderschülerIn « oder »Integrationskind « begründet. Adressierungsprozesse (Butler, 2009) wirken sich damit auf die ganze Persönlichkeit aus, was sich besonders in prospektiven Adressierungen zeigt. Eine Adressierung als sehr gute/r SchülerIn und das Integrieren dieser Adressierung in die eigene Identität und das Selbstkonzept führt in der Folge beispielsweise zu mehr Zutrauen in die eigene Leistung und zu real besse- 
rem Abschneiden bei Leistungsfeststellungen (vgl. dazu auch Rosenthal \& Jacobson, 1968).

Lehrpersonen beeinflussen die Subjektwerdung der SchülerInnen und damit auch deren Bildungskarrieren. Dies wird insbesondere im Kontext vorhandener Ungleichheit in unserem Bildungssystem diskutiert: Neben vielfach nachgewiesenen Risikofaktoren für erfolgreiche Bildungswege, die das soziale Umfeld betreffen (wie beispielsweise niedriger sozio-ökonomischer Hintergrund, niedrige Bildungsabschlüsse, Erwerbslosigkeit der Eltern, familiärer Migrationshintergrund; vgl. Bacher, 2009; Breit et al., 2016; Salchegger et al., 2016; Schreiner \& Breit, 2014; Wohlhart et al., 2016), sowie individuumsbezogene Faktoren (wie kognitive und physische Fähigkeiten, Geschlecht, bildungsbezogene Erfahrungen, Einstellungen und Verhaltensmerkmale; vgl. u. a. Bourdieu \& Passeron, 1971; Breit, 2016; Lange-Vester \& Teiwes-Kügler, 2006; Müller \& Pollak, 2010; Stamm, 2009; Stocké, 2009; Toferer et al., 2016) spielt auch das Bildungswesen (u.a. auch in Bezug auf darin verwurzelte exkludierende Strukturen und Praktiken) und dessen Akteurinnen und Akteure eine maßgebliche Rolle für die weiteren Bildungs- und Berufsbiografien von Kindern und Jugendlichen (vgl. u. a. Steiner et al., 2016; Statistik Austria, 2016; Wohlhart et al., 2016). Lehrpersonen und ihrem Handeln kommt eine besondere Bedeutung zu. Sie können einen großen Einfluss auf die Lernenden ausüben, daher sind neben ihren pädagogischen und diagnostischen Fähigkeiten auch ihre Beziehungsangebote, die von ihnen angebotene Unterstützung und Förderung sowie ihre subjektiven Theorien (bspw. Begabungstheorien) potenziell bedeutsam. Welche Rolle Lehrpersonen in individuellen Bildungskarrieren tatsächlich spielen, welche Einflussfaktoren für einen bestimmten Schüler bzw. eine bestimmte Schülerin im Einzelfall relevant werden und welche von (teilweise überraschend) geringem Stellenwert bleiben, hängt von den Grundorientierungen der SchülerInnen ab, wie in einer qualitativen Studie zu Bildungsaufstieg und vorzeitigem Bildungsausstieg dargelegt wird: Gerhartz-Reiter (2017) zeigt Erklärungsmuster für Bildungsverläufe und Gründe für die unterschiedliche Relevanz von einzelnen Faktoren in individuellen Laufbahnen auf und erklärt damit beispielsweise, warum manche SchülerInnen trotz zahlreicher Risikofaktoren hohe Bildungsabschlüsse erreichen, während andere, trotz Vorhandenseins vieler für Bildungslaufbahnen förderlicher Faktoren, das Bildungssystem vorzeitig verlassen. Es zeigt sich, dass bekannte Einflussfaktoren zwar Hürden bzw. Ressourcen für gelingende Bildungsverläufe darstellen und ihre Ausprägungen entsprechend Tendenzen anzeigen können, die für individuelle Bildungsverläufe ausschlaggebenden Aspekte aber die grundlegenden Ziele von SchülerInnen und der Beitrag des schulischen Feldes zur Erreichung dieser Ziele sind. Gängige Einflussfaktoren, gerade auch jene im schulischen Feld, so auch das Handeln von Lehrpersonen, sind dann von Bedeutung, wenn sie für die (Nicht-)Erreichung der grundlegenden Ziele eine Rolle spielen. Wenn beispielsweise Lehrpersonen einen Schüler, dessen wichtigstes Ziel sozia- 
ler Aufstieg ist, einerseits dabei unterstützen, seine schulischen Aufgaben zu bewältigen, andererseits aber auch in Bezug auf weiterführende Schul- und Ausbildungsmöglichkeiten nicht nur beraten, sondern je nach Bedarf auch tatkräftig unterstützen, kann das schulische Feld dem Schüler eine wichtige Stütze für das Erreichen seiner Ziele sein. Der Einfluss zahlreicher Risikofaktoren für das Erreichen höherer Bildung kann dadurch enorm verringert werden.

Je nach grundlegenden Zielen und individueller Ausgangslage muss das schulische Feld SchülerInnen also sehr unterschiedliche Unterstützung bieten. Dies zeigt den großen Stellenwert pädagogischer Diagnostik: Ohne das Erkennen der individuellen Ziele der SchülerInnen ist eine passgenaue Förderung und Unterstützung kaum möglich. Entsprechend groß ist der Bedarf einer pädagogischen Diagnostik, die Lehrpersonen ermöglicht, die Grundorientierungen der SchülerInnen präzise zu erkennen, um Bildungsverläufe bestmöglich begleiten und unterstützen zu können. Eine solche pädagogische Diagnostik hat wenig mit Kategorisierungen von SchülerInnen auf Basis ihrer sozialen, körperlichen, geistigen oder ökonomischen Ausgangslagen zu tun, sondern geht von der Frage aus, was eine Person möchte, was ihre grundlegenden Ziele sind, wovon sie sich abgrenzt und was sie als eigenes Potenzial sieht bzw. welche Unterstützung sie benötigt. Diese Fragen können selbstredend nicht nur auf Basis von Beobachtung und Leistungsfeststellung durch Lehrpersonen beantwortet werden, sondern brauchen einen Dialog mit SchülerInnen auf Augenhöhe, ihre gleichwertige Partizipation im diagnostischen Prozess.

\section{Pädagogische Diagnostik}

\subsection{Pädagogische Diagnostik als zentrale Aufgabe von Lehrkräften}

Während sonderpädagogische Diagnostik auf die Feststellung sonderpädagogischen Förderbedarfs und die Auswahl passender Förderungen bzw. Therapien für die jeweiligen SchülerInnen fokussiert, hat die pädagogische Diagnostik die Aufgabe, Lernvoraussetzungen und Lehr-Lernprozessen aller SchülerInnen zu analysieren. Darüber hinaus hat sie auch die Aufgabe, »die Zuweisung zu Lerngruppen oder zu individuellen Förderungsprogrammen [zu] ermöglichen sowie die mehr gesellschaftlich verankerten Aufgaben der Steuerung des Bildungsnachwuchses oder der Erteilung von Qualifikationen $[. .$.$] « (Ingenkamp \& Lissmann, 2008, S. 13). Pädagogische Diagnostik der$ Fähigkeiten von SchülerInnen stellt einen großen Teil des täglichen pädagogischen Handelns Lehrender dar und beeinflusst, in welcher Art und Weise Lehrende ihre SchülerInnen ansprechen, welche Lernangebote und -unterstützungen gesetzt und welche Bildungswegempfehlungen gegeben werden. Die OECD zählt pädagogische Diagnos- 
tik zu den zentralen Aufgaben von Lehrenden: »Teachers need diagnostic [...] tools that allow them to monitor and respond to what children are learning in order to ensure that they are acquiring a depth of understanding and knowledge « (OECD, 2012, S. 139).

Diagnostische Kompetenz wird zu den vier Basiskompetenzen für erfolgreichen Unterricht gezählt, wobei eine hohe Diagnosekompetenz mit darauf aufbauenden Strukturierungshilfen als besonders lernförderlich erachtet wird (Helmke, 2009, S. 121-143). Ebenso betont Hattie (2013) die Wichtigkeit des Zusammenhangs zwischen Lernerfolg und der Kenntnis des aktuellen Entwicklungsstands der SchülerInnen und das entsprechende Eingehen darauf durch die Lehrperson. Von besonderer Bedeutung erscheint dabei, dass pädagogische Diagnostik Teil der täglichen Arbeit von LehrerInnen ist und zugleich die Basis für ebendieses pädagogische Handeln darstellt - pädagogische Diagnostik ist damit eine auch in den Schulalltag eingebettete Aufgabe von LehrerInnen, wobei weiche (qualitative) bis harte (quantitative) Diagnoseinstrumente eingesetzt werden. Die Hauptaufgabe prozessorientierter pädagogischer Diagnostik besteht darin, für die Lernenden Hilfestellungen zu setzen bzw. für das eigene pädagogische Handeln richtungsweisende Entscheidungen zu treffen.

Forschung betont die dafür notwendige Diagnosekompetenz Lehrender. Nicht weniger wichtig ist jedoch die Einsicht in die Fehlbarkeit diagnostischer Urteile und in die Notwendigkeit einer kontinuierlichen Nachjustierung ebenso wie die eines mehrperspektivischen Blickes, um individuelle Färbungen von Urteilen möglichst gering zu halten. So ist es notwendig, die Perspektive von Kindern und Jugendlichen in die Diagnostik einzubeziehen, um die Aussagekraft pädagogischer Diagnosen zu verbessern und das darauf aufbauende pädagogische Handeln zu validieren und zu reflektieren. In diesem Sinne wird pädagogische Diagnostik als Unterstützungsmaßnahme für alle Kinder angestrebt, wobei die Informationsgewinnung als generelle Grundlage für pädagogisches Handeln, die Unterrichtsgestaltung und Bildungswegentscheidungen gesehen wird. Zweck ist nicht nur die Erstellung von Lehr- und Lernplänen bzw. die Planung der nächsten Unterrichtsschritte, sondern auch die Identifikation von Barrieren sowie deren Minderung und Behebung (J. Simon \& T. Simon, 2013). Dabei sollte das Augenmerk »auf wirksame Wege zur Unterstützung unterschiedlicher Gruppen von Lernenden gelegt werden, einschließlich der Lernenden mit besonderen Bedürfnissen und/oder Lernenden aus benachteiligten Verhältnissen « (Council of the European Union, 2014, S. 2). Dieses Ziel ist jedoch im aktuellen österreichischen wie deutschen Schulsystem zumindest zweitrangig, wenn eine Einteilung der Kinder in $\gg$ normal « und »abweichend $\ll^{2}$ erfolgt, da als Diagnostik wahrgenommene Handlungen häufig primär zum Zwecke der Ressourcenbeschaffung eingesetzt werden und das eigentliche Anliegen - die Unterstützung von Kindern und Jugendlichen in ihren Lernprozessen in den Hintergrund gerückt wird. So ist die Gefahr der Klassifizierung und Stigmati- 
sierung von Kindern und Jugendlichen durch pädagogische Diagnostik groß. Zentral für diesen Beitrag ist, dass traditionell die Entscheidungs- und Interpretationshoheit in diagnostischen Prozessen ausschließlich bei den Lehrenden liegt - SchülerInnen empfangen im Anschluss an diagnostische Prozesse beispielsweise Fördermöglichkeiten, sehen sich aber auch mit Selektionsmaßnahmen und Platzierungen konfrontiert. Im Gegensatz dazu wird im hier vorgestellten Projekt postuliert, dass Diagnostik ein gemeinsames Handeln von LehrerInnen und SchülerInnen sein sollte. Bereits Muckel (1996, S. 30) hat gefordert, dass » an Stelle von heimlichen Expost-Schlußfolgerungen aus den angestellten Verhaltensbeobachtungen [...] Schlußfolgerungs- und Erkenntnisprozesse des Diagnostikers in transparenter Weise in den diagnostischen Dialog mit den Betroffenen eingebracht werden « sollten. Da es darüber hinaus nicht möglich ist, von außen festzustellen, welche Motivationen, Orientierungsrahmen und Zielvorstellungen einen Menschen handlungsleitend prägen, ist es für Lehrende unerlässlich, SchülerInnen in diagnostische Prozesse einzubeziehen und durch einen Dialog die wesentlichen Aspekte für die notwendige Unterstützung sowie jene für die Planung weiteren Unterrichts gemeinsam zu erschließen. So sollten die DiagnostikerInnen auch nach Muckel (1996, S. 31) davon ausgehen, dass »die Probanden ein Wissen haben, das sie, die Diagnostiker, (noch) nicht kennen. Dieses Wissen macht sie zu Experten des zur Diskussion stehenden Problems und schafft ein zumindest potentielles Gegengewicht zur Macht des Diagnostikers «.

\subsection{Entwicklung einer partizipatorischen Diagnostik}

\subsubsection{Partizipatives Forschungsdesign im Kontext der Disability Studies}

Im Zentrum des vorgestellten Forschungsprojektes steht die Frage, wie pädagogische Diagnostik als Unterstützung für eigenverantwortliche und selbstbestimmte Bildungsprozesse von allen SchülerInnen genutzt werden kann. In der Pilotphase wird einerseits das Projekt partizipativer Diagnostik durchgeführt. Darin ist es zentral, dass SchülerInnen erkennen, welche Fähigkeiten sie haben und was sie brauchen, um gut lernen zu können. Sie sollen dies in einem Gespräch mit LehrerInnen artikulieren können, um im Anschluss daran gemeinsam mit Lehrenden Schritte für ihr weiteres Lernen, ihre Bildungswege und die Betreuung durch ihre LehrerInnen festlegen zu können. Im Rahmen des Projektes ist ein weiteres Ziel, diesen diagnostischen Prozess zu erforschen, um Möglichkeiten und Limitationen der konzipierten partizipativen pädagogischen Diagnostik zu erörtern. Dieses zweite Ziel wird ebenfalls partizipativ verfolgt und so findet die Beteiligung von SchülerInnen nicht nur im Rahmen des diagnostischen Prozesses, sondern auch im Rahmen der Forschung statt. 
Im Forschungsprojekt wird zwei zentralen Fragestellungen nachgegangen:

a) Wie können SchülerInnen durch pädagogische Diagnostik beim Erreichen selbstbestimmter Bildungsverläufe unterstützt werden?

b) Welche kollektiven Normen, ein- und ausgrenzenden Barrieren und Machtverhältnisse ermöglichen und begrenzen Partizipationsmöglichkeiten von SchülerInnen in ihren Bildungsprozessen?

Das Projekt verortet sich im Forschungsfeld der emanzipatorischen und partizipatorischen Disability Studies (Barnes, 2001). Das Prinzip der Disability Studies, »nichts über uns, ohne uns « (Hermes \& Rohrmann, 2006), ist grundlegend für das Forschungsdesign.

In dieser Studie werden Objekte pädagogischer Diagnostik zu handelnden Subjekten, indem sie selbst die Rolle der Diagnostizierenden und der Forschenden einnehmen: Ziel des Projekts ist es, nicht über, sondern mit den Betroffenen zu forschen, um deren persönliche Wahrnehmungen und Konstruktionen sichtbar zu machen und als wesentlichen Bestandteil der Forschung zu positionieren. Die teilnehmenden SchülerInnen sind als Ko-ForscherInnen an unterschiedlichen Stellen des Forschungsprozesses beteiligt. Nach Flieger (2003) kann die Involviertheit der betroffenen Personen in als partizipativ bezeichneten Forschungsvorhaben variieren - die Bandbreite reicht von der Rolle als InterviewpartnerInnen bis zu gänzlich gleichberechtigten Ko-ForscherInnen. In diesem Projekt sind die teilnehmenden SchülerInnen sowohl an der Erhebung als auch an der Analyse der Daten beteiligt, um sicherzustellen, dass Raum für die Artikulation der SchülerInnen geschaffen wird und ihre eigenen Ansichten und Deutungen einen zentralen Stellenwert erhalten. Allerdings wurden die Fragestellung, die aufgrund des Forschungsstandes sowie aufgrund der aktuellen Bedingungen im Schulsystem für die betroffenen Personen als brauchbar und relevant erachtet wird, und auch das vorläufige Forschungsdesign von den universitären ForscherInnen vorgegeben. An der Adaption bzw. Weiterentwicklung des Projekts sind die SchülerInnen dann aber wieder federführend beteiligt: Zum Abschluss erörtern die SchülerInnen Chancen und Hindernisse bezüglich Partizipation in der Schule und erarbeiten in diesem Kontext aus ihrer Sicht notwendige Veränderungen des Forschungsdesigns, indem sie ihre Erfahrungen mit der konzipierten Diagnostik diskutieren. So besteht für sie die Möglichkeit, als Ko-ForscherInnen das Projektdesign für künftige TeilnehmerInnen zu adaptieren. Darüber hinaus können sie nicht nur Anregungen aus dem Projekt für sich persönlich mitnehmen, sondern auch die in diesem Rahmen erworbenen Kenntnisse ihrer eigenen Fähigkeiten und Bedürfnisse, die zentral für das Gelingen ihrer weiteren Bildungslaufbahn sind.

Damit entspricht diese Forschungsarbeit auch dem von Flieger (2003, o.S.) formulierten Anspruch von Personen mit Behinderung an Forschung, nämlich dass diese 
»nützlich für ihr Leben sei und sich ihre Lebensqualität verbessere «. Dieser Grundsatz ist für das vorliegende Projekt zentral - der Nutzen der Kinder steht vor der wissenschaftlichen Verwertbarkeit. Im Mittelpunkt steht daher die aktive Teilnahme aller SchülerInnen am Prozess der pädagogischen Diagnostik ihrer Fähigkeiten und Bedürfnisse mit dem Ziel, ein höheres Maß an Autonomie in ihrem weiteren Bildungsweg und insbesondere ihren künftigen Bildungsentscheidungen zu erreichen. In diesem Forschungsprozess besteht die wesentliche Frage darin, wie SchülerInnen am besten erkennen und im Dialog mit ihren Lehrpersonen ausdrücken können, was sie brauchen, damit sie zu dem werden, was sie werden können und wollen, ohne durch Zuschreibungen hinsichtlich ihrer Fähigkeiten oder ihrer Unfähigkeit eingeschränkt zu werden.

Damit verbunden ist ein weiteres Anliegen dieses Projektes, und zwar dasjenige, bestehende Barrieren, gesellschaftlich wirkende Normen sowie wirkmächtige Diskurse und Machtstrukturen in der Zusammenarbeit mit den und insbesondere auch für die betroffenen SchülerInnen sichtbar zu machen und damit zu ihrer Reduzierung beizutragen.

\subsubsection{Konzeption des partizipativen Diagnose- und Forschungsprozesses}

Um den in Abschnitt 3.2.1 genannten Forschungsfragen nachzugehen, besteht das Forschungsprojekt im Wesentlichen aus vier Teilen, wobei sich die ersten drei Schritte auf die Durchführung der partizipativen pädagogischen Diagnostik beziehen und der vierte und abschließende Schritt den gesamten diagnostischen Prozess aus der Forschungsperspektive (mit Beteiligung der SchülerInnen) betrachtet: Die im folgenden dargelegten Schritte zielen auf die am Projekt beteiligten SchülerInnen mit den ihnen zur Verfügung stehenden Kompetenzen ab. Um die Teilhabe unterschiedlicher SchülerInnen mit verschiedenen Beeinträchtigungen zu gewährleisten, wird gegebenenfalls das Projektdesign adaptiert, wobei ausgehend von den unterschiedlichen Kindern und Jugendlichen die zentralen Fragestellungen leitend für die Adaption sind.

Im ersten Schritt der Studie verfassen SchülerInnen der Sekundarstufe I schriftliche Erinnerungsszenen zum Thema: »Hier konnte ich mein Bestes leisten.« Dabei wird nicht von einem Leistungsbegriff ausgegangen, der auf schulische/akademische Leistungen abzielt, sondern von einem, der weiter gefasst ist. Das heißt, dass auch Situationen des täglichen Lebens beschrieben werden können, wenn sie für die Kinder diese besondere Bedeutung haben.

Je nach individuellem Bedarf wird dabei Hilfestellung bzw. Unterstützung angeboten (in Bezug auf Kommunikation und Verschriftlichung oder durch zusätzliche visuelle erzählanregende Impulse etc.). Abhängig davon, welche Mittel zur Kommunikation den beteiligten SchülerInnen zur Verfügung stehen, werden diese auch in dem beschriebenen Prozess der pädagogischen Diagnostik eingesetzt (Niedick, 2016). 


\section{Erzählaufforderung:}

Beschreibe eine Situation, in der du dein Bestes leisten konntest.

Versuche über dieses Erlebnis eine Geschichte zu schreiben. Beschreibe und erzähle dabei so genau wie möglich. Schreibe alles, was dir dazu einfällt. Die folgenden Fragen können dir dabei helfen:

$>$ Was war das Beste, das du bisher leisten konntest - was ist dir besonders gut gelungen?

$>$ Wann konntest du dein Bestes leisten?

$>$ Wo ist dir das gelungen?

$>$ Wer war daran beteiligt?

Wie ist dir das überhaupt gelungen, so etwas zu leisten? Beschreibe dies so genau wie möglich!

$>\quad$ Was hat dir geholfen, dein Bestes leisten zu können?

$>$ Gab es Personen, die dabei für dich wichtig waren? Wie haben sie dich unterstützt?

Erzählungen werden als grundlegend für das menschliche Leben angesehen und spielen eine besondere Rolle in unserer Identitätskonstruktion. Erzählendes Wissen hilft uns, die Komplexität von Menschenleben zu verstehen, wobei das Geschichtenerzählen eine grundlegende menschliche Ausdrucksform ist. Aus diesem Grund ist das Design der Studie von der kollektiven Erinnerungsarbeit nach Frigga Haug (1990) inspiriert. Die verfassten Erinnerungsszenen sollen »ein gutes Zeugnis über herrschende Kultur und Ideologie, über Konstruktionsweisen, über Selbstwahrnehmung « ablegen und »einen Einblick [...] in das Forschungsfeld unter Einschluss der Subjekte « geben (Haug, 2003, S. 199), wobei eine kollektive Auswertung auch die Eingebundenheit der Subjekte in herrschende Strukturen sichtbar macht.

In einem zweiten Schritt werden die gesammelten Daten einige Tage später in von den universitären Forscherinnen geleiteten SchülerInnengruppen (jeweils ca. drei SchülerInnen) mithilfe einer Adaption der dokumentarischen Methode (Bohnsack, 2003) von den SchülerInnen selbst analysiert und interpretiert. Das Ziel dieser Methode ist es, implizites Wissen und (kollektive) Orientierungsrahmen zu rekonstruieren. Den Orientierungsrahmen einer Person explizit zu machen hilft, ihre Handlungslogik nachzuvollziehen, ihre Bedürfnisse zu verstehen und zu erkennen, was sie anstreben und erreichen wollen. Diese Informationen sind entscheidend für eine maßgeschneiderte Unterstützung einzelner SchülerInnen, um ihr Lernen und ihre schulischen Entscheidungen und Karrieren bestmöglich zu unterstützen (Gerhartz-Reiter, 2017). In diesem Prozess stehen den SchülerInnen die Leiterinnen des Forschungsprojektes als Ko-Forschende zur Seite, der Auswertungsprozess erfolgt im Team. Er beginnt mit der Analyse und Interpretation einer Geschichte, gefolgt 
von einer komparativen Vorgehensweise. Dabei erarbeiten die Kinder entlang von unterstützenden Fragen positive und negative Gegenhorizonte sowie das Enaktierungspotenzial des jeweiligen Autors/der jeweiligen Autorin. Durch das Festhalten der gemeinsam erarbeiteten Ergebnisse soll eine Basis für das Gespräch mit der Lehrkraft entwickelt werden.

\section{Analyse mit SchülerInnen in Kleingruppen im Rahmen des dialogischen Diagnoseprozesses}

Die SchülerInnen beantworten zuerst in Einzelarbeit Reflexionsfragen zu einzelnen Geschichten (Was ist der Person in der Geschichte besonders gut gelungen? Was gefällt dir an der Geschichte? Was findet die Person gut? Wobei braucht die Person Unterstützung?).

Im Anschluss erfolgt die Arbeit in der Gruppe mit einer komparativen Vorgehensweise. Wichtig ist dabei, die unterstützenden Fragen niederschwellig in der Sprache der SchülerInnen zu stellen. Die Auswahl einzelner Fragen und Themenbereiche ist abhängig von Situation und Gesprächsverlauf. Auf jeden Fall kommen Fragen zum Einsatz, die dazu dienen, die Eckpunkte des Orientierungsrahmens (positive Gegenhorizonte - negative Gegenhorizonte - Enaktierungspotenzial) zu erarbeiten. Ziel ist es, gemeinsam herauszufinden, was die Person möchte, was ihre Ziele sind und was sie braucht (von sich, von anderen, in Lernsituationen etc.), um diese zu erreichen (Was ist der Person in der Geschichte gut gelungen? Was findet die Person (nicht) gut? Was mag die Person (nicht)? Was ist schwierig in der Situation? Wie schafft die Person es, die Aufgabe zu lösen? Was/Wer hilft ihr dabei? Was kann die Person? Woher kann sie das? Was ist der Person wichtig? Wie möchte die Person (nicht) sein? Was braucht die Person von sich/von anderen?).

Die Ergebnisse werden im Gesprächsverlauf auf Kärtchen festgehalten, die die SchülerInnen mit in das Gespräch mit den LehrerInnen nehmen. Am Ende besitzen alle SchülerInnen Kärtchen mit Antworten zu folgenden Fragen: Was finde ich gut/Was ist mir wichtig? Was brauche ich? Welche Ziele/Vorbilder habe ich?

Als Vorbereitung auf die Gespräche verfassen die LehrerInnen (parallel zu ihren SchülerInnen) ebenfalls Geschichten in Form eines Briefes an die SchülerInnen. Der Schreibauftrag lautet: »Beschreibe eine Situation in der ... sein/ihr Bestes leisten konnte.« Diese Geschichten bearbeiten sie selbst unter ähnlichen Fragestellungen wie die SchülerInnen und reflektieren damit ihre Sicht auf das Kind. Die LehrerInnen stellen auf Basis dieser Geschichte weitere Überlegungen zu den eigenen subjektiven Theorien und Überzeugungen an (Was finde ich als SchreiberIn gut? Was stelle ich positiv dar, was negativ? Wie möchte ich als LehrerIn gerne sein? Welche Eigenschaften finde ich gut/nicht gut? Was sind für mich »ideale «/»schwierige « SchülerInnen?). 
Im dritten Schritt des Forschungsprojekts wird Raum geschaffen für dialogische diagnostische Prozesse unter Einbezug von Lehrenden und Lernenden (sowie je nach Anlassfall auch Eltern): LehrerIn und SchülerIn erarbeiten entlang eines vorbereiten Gesprächsleitfadens eine gemeinsame Diagnose zu Lernstand, -voraussetzungen und -bedürfnissen, in der die Erkenntnisse der Schülerin/des Schülers ebenso berücksichtigt werden wie die auf Beobachtungen, Leistungsfeststellungen und Elterngesprächen basierenden Einsichten der Lehrkraft. Auf Basis dieser gemeinsam erarbeiteten Diagnose erfolgt die kooperative Planung weiterer Schritte (Unterstützung von Lernprozessen, Bildungswegentscheidungen etc.).

\section{Gesprächsleitfaden}

Die/Der SchülerIn liest die eigene Geschichte vor und erklärt, was sich darin in Bezug auf Fähigkeiten und Bedürfnisse zeigt, und bringt eventuell zusätzliche Informationen ein. Es besteht für die Lehrenden die Möglichkeit nachzufragen.

Anschließend liest die Lehrkraft ihre Geschichte vor, erläutert ebenfalls ihre Annahmen in Bezug auf Fähigkeiten und Bedürfnisse der Schülerin/des Schülers, wobei wiederum die Möglichkeit des Nachfragens besteht.

Gemeinsam erarbeiten SchülerInnen und Lehrende im Anschluss daran, wo sich Übereinstimmungen/Unterschiede zeigen. Sie überlegen von den Bedürfnissen und Ressourcen (der Schülerin/des Schülers, der Lehrerin/des Lehrers, der Schule) ausgehend, wie eine passende Lernunterstützung für die SchülerInnen aussehen könnte, und beschließen konkrete Pläne für die nahe Zukunft.

Abschließend erfolgt eine Rückschau der SchülerInnen und universitären Forscherinnen auf den gesamten diagnostischen Prozess. Im Rahmen einer Gruppendiskussion werden die Wirkweisen, Möglichkeiten und Hindernisse der konzipierten partizipatorischen Diagnostik analysiert und reflektiert. Bei der Erörterung von grundsätzlichen Chancen und Grenzen partizipativer Prozesse in der Schule werden geltende Normensysteme und wirkmächtige Diskurse expliziert und diskutiert.

Insgesamt fokussiert die Studie ein Forschungsdesign, das für die Teilhabe aller SchülerInnen geeignet ist, auch für jene, denen mittels eines sonderpädagogischen Fördergutachtens temporär oder dauerhaft Fähigkeiten abgesprochen werden, bei schulischer pädagogischer Diagnostik mitwirken zu können. Das Modell kann und soll in der Folge von Lehrenden selbstständig eingesetzt werden, um einerseits ihren SchülerInnen echte Teilhabe an ihren Bildungskarrieren zu ermöglichen und andererseits auf Basis der zusätzlichen Informationen und Sichtweisen Rückschlüsse für ihr konkretes pädagogisches Handeln zu ziehen und dieses fokussierter auf die Bedürfnisse der SchülerInnen ausrichten zu können. 


\section{Konklusion}

Die hier vorgestellte Konzeption einer befähigenden, partizipativen Diagnostik dient dazu, alle SchülerInnen bei ihrer Entwicklung hin zu aktiven GestalterInnen ihrer Lernund Bildungsprozesse zu begleiten. Die Studie nutzt daher ein Forschungsdesign, das alle SchülerInnen - auch diejenigen, denen durch sonderpädagogische Fördergutachten vorübergehend oder dauerhaft bestimmte Fähigkeiten zugeschrieben oder abgesprochen werden - in Forschungsprozessen zur pädagogischen Diagnostik in Schulen als aktive TeilhaberInnen sieht. Da in der pädagogischen Diagnostik »das Augenmerk auf wirksame Wege zur Unterstützung unterschiedlicher Gruppen von Lernenden gelegt werden [sollte], einschließlich der Lernenden mit besonderen Bedürfnissen und/oder Lernenden aus benachteiligten Verhältnissen « (Council of the European Union, 2014, S. 2), ist das Forschungsdesign so angelegt, dass im Bedarfsfall dazu unterschiedlichste Unterstützungsmöglichkeiten angeboten werden. Durch den Prozess sollen bestehende Barrieren, gesellschaftliche Normen sowie wirkmächtige Diskurse und Machtstrukturen sichtbar gemacht und damit zu deren Reduzierung beigetragen werden. Dieses Projekt verfolgt damit auch das emanzipatorische Ziel, mehr »Kontrolle, Macht und Entscheidungsgewalt in [die] Hände von [Menschen] mit Behinderung « zu legen (Flieger, 2009, o. S.).

Die konzipierte partizipatorische pädagogische Diagnostik wurde in einer Pilotphase mit SchülerInnen aus zwei Integrationsklassen durchgeführt. Dabei konnten sowohl unterschiedliche Limitationen als auch verschiedene Möglichkeiten der Weiterarbeit exploriert werden.

\subsection{Limitationen}

In der Pilotphase des Projektes zeigten sich bestimmte Voraussetzungen, die für das Gelingen des diagnostischen Prozesses notwendig erscheinen. Von grundlegender Wichtigkeit erwiesen sich die Haltungen und das Menschenbild der LehrerInnen. So ist aufseiten der Lehrenden die Bereitschaft zur Partizipation der SchülerInnen und zur Adaption des Urteils über sie notwendig. SchülerInnen als gleichwertige, nach größtmöglicher Autonomie strebende Individuen zu sehen, zählt zu den Grundbedingungen für das Gelingen des Prozesses.

Es zeigte sich, dass eine punktuelle Durchführung des diagnostischen Prozesses den Einfluss und die Wichtigkeit der Rolle der SchülerInnen nur marginal verstärkt, wenngleich bereits eine einmalige Anwendung die Korrektur des Urteils der Lehrenden und eine größere Sensibilität bezüglich der eigenen Urteilsakkuratheit zur Folge haben kann. Insgesamt lässt sich jedoch die Notwendigkeit einer Adaption des Modells für einen regelmäßigen/wiederholten Einsatz folgern. 
Innerhalb der Analysegespräche in Kleingruppen erwiesen sich Hilfestellungen durch die Diskussionsleitenden zur Fokussierung und Artikulation als notwendig. Diese Hilfestellungen dürften sich bei regelmäßiger Verwendung minimieren lassen. Kritisch anzumerken bleibt, dass trotz bereits vorgenommener Adaptionen keine gleichberechtigte Nutzungsmöglichkeit für alle SchülerInnen gegeben ist, da die verbale Ausdrucksmöglichkeit grundlegend ist.

Trotz der Möglichkeiten zur Überwindung vorgefasster Meinungen und subjektiver Theorien der Lehrkräfte zu einzelnen SchülerInnen besteht die Gefahr, dass keine Erweiterung der bisherigen Perspektive eintritt, sondern durch selektive Wahrnehmung bereits fokussierte Aspekte eine vermeintliche Bestätigung erhalten und dadurch ein weiteres Festschreiben des bisherigen Fremdbildes erfolgt. Der angestrebte Austausch von SchülerInnen und LehrerInnen sollte im Idealfall zu einer Ergänzung bzw. Erweiterung der jeweiligen Perspektiven, eventuell zu einer Annäherung der beiden Positionen, jedenfalls aber - auch bei möglichem Dissens zwischen SchülerIn und LehrerIn - zumindest zu einer kritischen Reflexion des eigenen Bildes führen. Insgesamt zeigen die Ergebnisse, dass, wie auch Muckel (1996, S. 31) betont, »die Qualität und Gestaltung der diagnostischen Beziehung [...] die Qualität und Gültigkeit der dort gewonnenen Erkenntnisse mit[konstituiert] $\ll$.

\subsection{Ausblick}

Zentral erscheint die Erkenntnis, dass Zuschreibungsprozessen ein identitätsstiftender und transformierender Charakter innewohnt, wodurch sie ein »Ort der Macht « (Butler, 2009, S. 11) sind. Damit stellen diagnostische Zuschreibungsprozesse ein gewisses Risiko dar, mit ihnen sind jedoch auch viele Chancen verbunden (Reisenauer \& UlseßSchurda, 2018).

Die Pilotphase zeigt, dass durch die konzipierte partizipative Diagnostik zum einen SchülerInnen die Erkenntnis und Artikulation ihrer Fähigkeiten und Bedürfnisse ermöglicht wird, zum anderen eine Perspektivenerweiterung der Lehrenden erfolgen kann. Ebenso kann daraus eine Bewusstwerdung über die Beschränktheit und Subjektivität des eigenen Fremdurteils resultieren. Damit wird - wie angestrebt - eine Möglichkeit zur Erhöhung der Treffsicherheit pädagogischer Diagnostik geboten. Darüber hinaus zeigt sich eine veränderte Wahrnehmung der SchülerInnen untereinander. Durch die Arbeit in den Kleingruppen lenken die Kinder und Jugendlichen ihre Aufmerksamkeit auf die einzelnen Gruppenmitglieder, ihre individuellen Stärken und Bedürfnisse. Dadurch erfolgt eine Sensibilisierung, die möglicherweise einen positiven Einfluss auf das weitere Leben, Arbeiten und Lernen im Klassenverband haben kann. 
Die Teilhabe von SchülerInnen an diagnostischen Prozessen zeigt sich damit als gewinnbringend für alle Beteiligten. Das Modell muss jedoch dahingehend erweitert/überarbeitet werden, dass die Arbeit der Lernenden weniger an die sprachliche Ausdrucksfähigkeit der SchülerInnen gekoppelt ist und so niemand in der Teilhabe beschränkt wird.

Die Ergebnisse des Projekts sind nicht nur auf der individuellen Ebene, sondern auch auf einer gesamtgesellschaftlichen Ebene und als Beitrag für eine inklusive Schule zu sehen. Mit den Ergebnissen wird ferner ein Beitrag zur weiteren Professionalisierung von Lehrenden besonders im Bereich pädagogischer Diagnostik geleistet.

Da eine wiederholte Anwendung wesentlich für die positive Wirkung des Modells ist, werden Möglichkeiten der Implementierung in einer Versuchsschule diskutiert. Weitere geplante Schritte umfassen die Entwicklung eines Modells, das im Verfahren der Eingangsdiagnostik von SchülerInnen in der Primarstufe (vor Feststellung des sonderpädagogischen Förderbedarfs) genutzt werden soll. Darüber hinaus wird eine Variante für inklusive Schulsettings entworfen, die kontinuierliche inklusionsorientierte Diagnostik anstelle der Festschreibung sonderpädagogischen Förderbedarfs ermöglichen soll.

\section{Anmerkungen}

1 Ziel dieser Untersuchung war ein besseres Verständnis der Gründe für Bildungs(miss)erfolg, um eine möglichst passende Unterstützung individueller Bildungsverläufe zu ermöglichen, Bildungsaufstieg zu fördern und vorzeitigen Bildungsausstieg einzudämmen. Um der Frage nachzugehen, wie Bildungskarrieren gelingen, wurden in 22 biografisch-narrativen Interviews junge Erwachsene befragt. Die Auswertung erfolgte mittels der dokumentarischen Methode (Bohnsack, 2003).

2 Eine zentrale Aufgabe pädagogischer Diagnostik ist die Erstellung eines Gutachtens, in dem sonderpädagogischer Förderbedarf festgestellt wird und Kinder damit einem speziellen Schultyp bzw. Integrationsklassen zugewiesen werden.

\section{Literatur}

Bacher, J. (2009). Soziale Ungleichheit, Schullaufbahn und Testleistungen. In B. Suchań, C. WallnerPaschon \& C. Schreiner (Hrsg.), PIRLS 2006. Die Lesekompetenz am Ende der Volksschule - Österreichischer Expertenbericht. Graz: Leykam. https://www.bifie.at/buch/875/6 (24.03.2015).

Balzer, N. \& Ricken, N. (2010). Anerkennung als pädagogisches Problem - Markierungen im erziehungswissenschaftlichen Diskurs. In A. Schäfer \& C. Thompson (Hrsg.), Anerkennung (S. 35-87). Paderborn: Schöningh (Pädagogik Perspektiven).

Barnes, C. (2001). »Emancipatory« disability research: Project or process? http://disability-studies.leeds.ac. uk/files/library/Barnes-glasgow-lecture.pdf (26.3.2018).

Bohnsack, R. (2003). Rekonstruktive Sozialforschung (8. Aufl.). Opladen: Leske + Budrich.

Bourdieu, P. \& Passeron, J.-C. (1971). Die Illusion der Chancengleichheit. Stuttgart: Ernst Klett Verlag. 
Breit, S. (2016). Überschneidungen zwischen den Spitzen- bzw. Risikogruppen in Naturwissenschaft, Lesen und Mathematik. In B. Suchań \& S. Breit (Hrsg.), PISA 2015. Grundkompetenzen am Ende der Pflichtschulzeit im internationalen Vergleich (S. 73-76). Graz: Leykam.

Breit, S., Bruneforth, M. \& Schreiner, C. (Hrsg.). (2016). Standardüberprüfung 2015 Deutsch, 4. Schulstufe. Bundesergebnisbericht. Salzburg: BIFIE.

Butler, J. (2003). Kritik der ethischen Gewalt. Adorno-Vorlesungen 2002, Institut für Sozialforschung an der Johann Wolfgang Goethe-Universität, Frankfurt am Main. Frankfurt am Main: Suhrkamp.

Butler, J. (2009). Die Macht der Geschlechternormen und die Grenzen des Menschlichen. Frankfurt am Main: Suhrkamp.

Council of the European Union (2014). Schlussfolgerungen des Rates vom 20. Mai 2014 zu wirksamer Lehrerausbildung. http://eur-lex.europa.eu/legal-content/DE/TXT/PDF/?uri=CELEX:52014XG0614(05)\& from $=\mathrm{DE}(15.01 .2018)$.

Flieger, P. (2003). Partizipative Forschungsmethoden und ihre konkrete Umsetzung. In G. Hermes \& S. Köbsell (Hrsg.), Disability Studies in Deutschland - Behinderung neu Denken. Dokumentation der Sommeruni (S. 200-204). Kassel: bifos. http://bidok.uibk.ac.at/library/flieger-partizipativ.html (15.01.2018).

Flieger, P. (2009). Partizipatorische Forschung. Wege zur Entgrenzung der Rollen von Forschrinnen und Beforschten. In J. Jerg, K. Merz-Atalik, R. Thümmler \& H. Tiemann (Hrsg.), Perspektiven auf Entgrenzung. Erfahrungen und Entwickungsprozesse von Inklusion und Integration (S. 159-171). Bad Heilbrunn: Klinkhardt. http://bidok.uibk.ac.at/library/flieger-partizipatorisch.html (15.01.2018).

Gerhartz-Reiter, S. (2017). Erklärungsmuster für Bildungsaufstieg und Bildungsausstieg. Wie Bildungskarrieren gelingen. Wiesbaden: VS.

Hattie, J. (2013). Lernen sichtbar machen. Überarbeitete deutschsprachige Ausgabe von »Visible Learning«. Unter Mitarbeit von Wolfgang Beywl und Klaus Zierer (3., erweit. Aufl. mit Index und Glossar). Baltmannsweiler: Schneider Hohengehren.

Haug, F. (1990). Erinnerungsarbeit. Hamburg: Argument-Verlag.

Haug, F. (2003). Erinnerung an Lernen. Journal für Psychologie, 11(2), 194-213. http://nbnresolving.de/ urn:nbn:de:0168-ssoar-17421 (07.06.2016).

Hegel, G.W.F. (1970 [1806/07]). Phänomenologie des Geistes (Werke Band 3). Frankfurt am Main: Suhrkamp.

Helmke, A. (2009). Unterrichtsqualität und Lehrerprofessionalität: Diagnose, Evaluation und Verbesserung des Unterrichts (3. Aufl.). Seelze-Velber: Klett/Kallmeyer.

Helsper, W. (2000). Antinomien des Lehrerhandelns und die Bedeutung der Fallrekonstruktion - Überlegungen zu einer Professionalisierung im Rahmen universitärer Lehrerausbildung. In E. Cloer, D. Klika \& H. Kunert (Hrsg.), Welche Lehrer braucht das Land? (S. 142-177). Weinheim: Juventa.

Hermes, G. \& Rohrmann, E. (Hrsg.). (2006). Nichts über uns - ohne uns! Disability Studies als neuer Ansatz emanzipatorischer und interdisziplinärer Forschung über Behinderung. Neu-UIm: AG SPAK Bücher.

Ingenkamp, K. \& Lissmann, U. (2008). Lehrbuch der Pädagogischen Diagnostik. Weinheim, Basel: Beltz Verlagsgruppe.

Lange-Vester, A. \& Teiwes-Kügler, C. (2006). Die symbolische Gewalt der legitimen Kultur. Zur Reproduktion ungleicher Bildungschancen in Studierendenmilieus. In W. Georg (Hrsg.), Soziale Ungleichheit im Bildungssystem. Eine empirische-theoretische Bestandsaufnahme (S. 55-92). Konstanz: UVK.

Lipowski, F. (2006). Auf den Lehrer kommt es an. Empirische Evidenzen für Zusammenhänge zwischen Lehrerkompetenzen, Lehrerhandeln und dem Lernen der Schüler. Zeitschrift für Pädagogik, (52), 51. Beiheft, 47-70.

Muckel, P. (1996). Qualitative Psychodiagnostik: Überlegungen zu einer Theorie subjektorientierter Psychodiagnostik. Journal für Psychologie, 4(4), 28-36. http://nbn-resolving.de/urn:nbn:de:0168 -ssoar-29304 (18.07.2018). 
Müller, W. \& Pollak, R. (2010). Weshalb gibt es so wenige Arbeiterkinder in Deutschlands Universitäten? In R. Becker \& W. Lauterbach (Hrsg.), Bildung als Privileg. Erklärungen und Befunde zu den Ursachen der Bildungsungleichheit (S. 305-344). Wiesbaden: VS.

Niedick, I. (2016). Wer nicht fragt, bekommt keine Antworten - Interviewtechniken unter besonderen Bedingungen. Zeitschrift für Inklusion, 4/2016. https://www.inklusion-online.net/index.php/ inklusion-online/article/view/323 (15.3.2018).

OECD (2012). Equity and quality in education. Supporting disadvantaged students and schools. Paris: OECD Publishing.

Ophuysen, S. van \& Behrmann, L. (2015). Die Qualität pädagogischer Diagnostik im Lehrerberuf - Anmerkungen zum Themenheft »Diagnostische Kompetenzen von Lehrkräften und ihre Handlungsrelevanz«. Journal for educational research online, 7(2), 82-98.

Pfahl, L. \& Köbsell, S. (2014). Was sind eigentliche Disability Studies? Forschung \& Lehre, (7/2014), 554-555. http://bidok.uibk.ac.at/library/pfahl-disability.html (15.03.2018).

Reisenauer, C. \& Ulseß-Schurda, N. (2018). Anerkennung in der Schule. Über Anlässe, Abläufe und Wirkweisen von Adressierungen. Bern: Hep-Verlag.

Ricken, N. (2009). Über Anerkennung. Spuren einer anderen Subjektivität. In N. Ricken, H. Röhr, J. Ruhloff \& K. Schaller (Hrsg.), Umlernen. Festschrift für Käthe Meyer-Drawe (S. 75-91). Paderborn: Fink.

Rosenthal, R. \& Jacobson, L. (1968). Pygmalion in the classroom. New York: Holt, Rinehart \& Winston.

Salchegger, S., Wallner-Paschon, C., Schmich, J. \& Höller, I. (2016). Kompetenzentwicklung im Kontext individueller, schulischer und familiärer Faktoren. In PISA 2015. Grundkompetenzen am Ende der Pflichtschulzeit im internationalen Vergleich (S. 77-99). Graz: Leykam.

Schreiner, C. \& Breit, S. (Hrsg.). (2014). Standardüberprüfung 2013 Englisch, 8. Schulstufe. Bundesergebnisbericht. Salzburg: BIFIE.

Sichler, R. (1996). Diagnostische Kompetenz und reflektierte Praxis: psychologische Diagnostik als dialogischer Prozeß. Journal für Psychologie, 4(4), 18-27. http://nbn-resolving.de/urn:nbn:de:0168 -ssoar-29314 (18.07.2018).

Simon, J. \& Simon, T. (2013). Inklusive Diagnostik - Wesenszüge und Abgrenzung von traditionellen »Grundkonzepten« diagnostischer Praxis. Eine Diskussionsgrundlage. Zeitschrift für Inklusion, (4). https://www.inklusion-online.net/index.php/inklusion-online/article/view/194 (15.03.2018).

Stamm, M. (2009). Begabte Minoritäten. Wiesbaden: VS.

Statistik Austria (2016). Bildung in Zahlen 2014/15. Schlüsselindikatoren und Analysen. Wien: Statistik Austria.

Steiner, M., Pessl, G. \& Bruneforth, M. (2016). Früher Bildungsabbruch - Neue Erkenntnisse zu Ausmaß und Ursachen. In M. Bruneforth, F. Eder, K. Krainer,C. Schreiner, A. Seel \& C. Spiel (Hrsg.), Nationaler Bildungsbericht Österreich 2015. Fokussierte Analysen bildungspolitischer Schwerpunktthemen, Band 2 (S. 175-220). Graz: Leykam.

Stocké, V. (2009). Adaptivität oder Konformität? Die Bedeutung der Bezugsgruppe und der Leistungsrealität der Kinder für die Entwicklung elterlicher Bildungsaspirationen am Ende der Grundschulzeit. In J. Baumert, K. Maaz \& U. Trautwein (Hrsg.), Bildungsentscheidungen. Zeitschrift für Erziehungswissenschaft, Sonderheft 12, 257-281.

Toferer, B., Höller, I., Schmich, J. \& Suchań, B. (2016). Kompetenzen der Schüler/innen in Lesen. In PISA 2015. Grundkompetenzen am Ende der Pflichtschulzeit im internationalen Vergleich (S. 57-65). Graz: Leykam.

Wohlhart, D., Böhm, J., Grillitsch, M., Oberwimmer, K., Soukup-Altrichter, K. \& Stanzel-Tischler, E. (2016). Die österreichische Volksschule. In M. Bruneforth, F. Eder, K. Krainer, C. Schreiner, A. Seel \& C. Spiel (Hrsg.), Nationaler Bildungsbericht Österreich 2015. Fokussierte Analysen bildungspolitischer Schwerpunktthemen, Band 2 (S. 17-58). Graz: Leykam. 


\section{Die Autorinnen}

Sabine Gerhartz-Reiter, MMag., BA PhD, ist an der Universität Innsbruck am Institut für LehrerInnenbildung und Schulforschung tätig. Ihre Arbeitsschwerpunkte sind: Ungleichheit im Bildungswesen, Bildungskarrieren, pädagogisches Handeln und LehrerInnenbildung.

Kontakt: sabine.gerhartz-reiter@uibk.ac.at

Cathrin Reisenauer, MMag. Dr., ist ebenfalls am Institut für LehrerInnenbildung und Schulforschung der Universität Innsbruck beschäftigt. Ihre Arbeitsschwerpunkte sind: Inklusion im Bildungswesen, pädagogisches Handeln, Anerkennung sowie Zuschreibungsprozesse.

Kontakt: cathrin.reisenauer@uibk.ac.at 